\title{
Real-Time Under Load Electrochemical Impedance Spectroscopy (EIS) Analysis and Modeling
}

\author{
H. Leduc, R. Okamura, E. Kyeyune-Nyombi Jr., K. Huynh, and S. Chung \\ ReJoule, Inc. Stanton, California 92841, USA
}

\begin{abstract}
As renewable energy usage continues to rise, the need for reliable energy storage solutions also grows. Today, battery systems are expected to last for several years. The challenge comes in monitoring battery safety and degradation. A major limitation in tracking the performance of battery cells is the lack of relevant measurements. Electrochemical impedance spectroscopy (EIS) has been used to study underlying battery phenomena. However, EIS is typically only performed on batteries which have been at rest for long periods of time - a requirement that is impractical in today's energy storage systems. Here, the usability of EIS in real-time is investigated on batteries subjected to different current loads. Limitations in signal processing and electrochemistry for EIS taken under load are also discussed and its usability is demonstrated in estimating the internal cell temperature in extreme conditions. This broadens the use of EIS for commercial applications.
\end{abstract}

\section{Introduction}

As clean energy and sustainable transportation usage rises sharply across the world, accurate and accessible battery health monitoring is of growing importance. This is particularly true in electric vehicle (EV) and energy storage system (ESS) applications, where a large number of battery cells make up a large-format battery pack that has an operational lifetime of $10+$ years. Battery health monitoring in large-format battery packs is especially challenging, for two major reasons: 1) electrochemical cells degrade nonuniformly, and 2) available battery diagnostic test techniques are impractical to perform on large battery systems from a cost and resource perspective. This paper focuses on the lack of sufficient battery diagnostic tests and proposes an alternative method for obtaining relevant metrics in real-time environments using a technique called electrochemical impedance spectroscopy (EIS).

Battery pack performance and health is often characterized using only cell voltages and easy-to-access surface temperatures because these are often the only available field measurements. From just these parameters, online battery algorithms are tasked to track critical performance indicators such as battery state-of-charge (SOC), state-of-health $(\mathrm{SOH})$, and state-of-power (SOP) of numerous individual cells - all of which diverge over time (1-2). This is especially challenging because many environmental and utilization factors affect cell terminal voltage measurements, including fluctuations in temperature, changing charge and discharge currents, and cell balancing. Different variations of nonlinear state estimators like extended kalman filtering are commonly used 
to track performance indicators and account for changing environmental factors (3-4). However, the lack of available real-time metrics tends to lead to increased computation in order to achieve acceptable accuracy. Other approaches such as physics-based modeling have also been examined (5-7). However, these complex models require computational resources that are simply not practical for real-time applications, especially for large-format battery systems.

EIS is a powerful technique for analyzing various electrochemical systems. One common application includes using EIS as a non-invasive means of studying the underlying electrochemical phenomena in battery cells. The battery's alternating current impedance (ACI), which is output from an EIS test, has been shown to have a strong correlation with SOC, battery degradation, and temperature (8-12). Recently, various technologies have emerged that allow for the real-time application of EIS in battery management systems (BMS) (13-15). Unfortunately, EIS is typically performed in static conditions, making EIS a challenge to implement in real-time applications.

In a study by J.P Diard et al, EIS measurements were taken on Nickel-Cadmium (NiCd) and Lead Acid cells that were under constant current loads (16). The results of the investigation showed that the EIS performed under small loads were applicable in high-capacity, low-impedance systems.

In this paper, the usability of EIS on lithium ion (Li-Ion) batteries under different current loads is explored and the use of EIS under load for estimating internal cell temperature is demonstrated.

\section{Background: Electrochemical Impedance Spectroscopy}

EIS consists of injecting an excitation voltage or current signal into a device under test (DUT) over a range of frequencies, then measuring a response current or voltage signal, respectively. The impedance of the DUT can then be calculated by simply using Ohm's Law.

$$
Z(j \omega)=\frac{V(j \omega)}{I(j \omega)}
$$

The excitation signal is in the form of sinusoidal waves at a given frequency and the response is generally analyzed using some form of Fourier transform. The excitation and response signals are represented in figure 1 on the battery current-voltage (i-V) characteristic curve. The impedance response is a complex number. The output of an EIS measurement, the ACI spectrum, is the overall impedance response over the frequency range. The ACI is generally displayed into two formats:

- a Bode plot comprised of two plots, the magnitude and the phase response as a function of the frequency and

- a Nyquist plot consists of a single plot of the imaginary part of the impedance as a function of its real part. Generally, the frequency is not displayed on the graph.

In this paper, the Nyquist plot is preferred over the Bode plot as it is a powerful tool to aggregate and analyze electrochemical phenomena and materials' global structure and 
stability. It is important to note that the interpretation of a Nyquist plot can legitimately be done only when both axes are on the same scale. An example of a Nyquist plot is displayed with a few marked frequencies in figure 1.
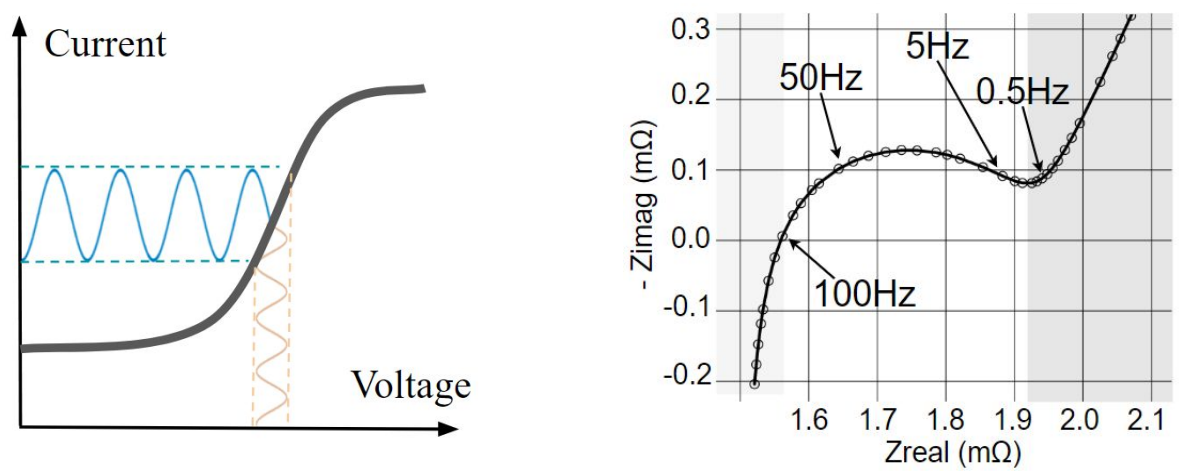

Figure 1. (left) The typical i-V characteristic of a battery, (right) Nyquist plot of a Li-ion battery.

When analyzing an EIS spectrum of a Li-ion battery, the key components are separated by frequency ranges:

- At higher frequencies, inductive characteristics and the effects of the electrolyte resistance drive the ACI spectra, as highlighted in light gray in figure 1.

- In the intermediate frequency range, charge transfer resistance, double layer capacitance and solid electrolyte interface (SEI) can be observed

- In the lower frequency range, the diffusion phenomena inside the electrodes can be observed, in darker grey on the Nyquist in plot figure 1.

Typically, EIS can only be performed on systems that are linear, causal, and stable:

- Causality in battery systems can be assumed as the response behavior is sufficiently finite.

- Linearity can be assumed by using a relatively small excitation signal. As illustrated on figure 1 , the (i-V) characteristic of a battery is not linear but when the input signals are small enough, the linear approximation is valid. This condition is generally verified with a good signal-to-noise ratio when the perturbation current is around $10 \%$ of the battery capacity.

- Stability is often the most difficult criterion to satisfy as it often requires cells to be held at rest for a long time. This allows for the materials to rest and to be stable so the mechanisms of interest can be properly studied, especially at low frequencies. Unfortunately, such extended rest periods are often not feasible in a real-time application, especially in large-format battery packs.

EIS is a very sensitive measurement as the input and output signals must remain very small to verify the linearity criterion. Also, EIS is very sensitive to temperature. The materials' structure and kinetics highlighted above are all dependent upon temperature. Figure 2 displays the evolution of ACI measurements with respect to temperature. Consequently, EIS measurements can legitimately be utilized as internal cell temperature indicators. 


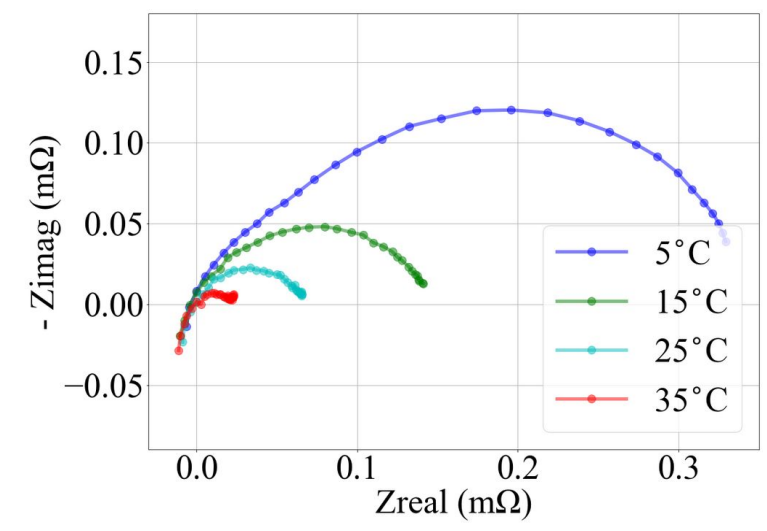

Figure 2. Normalized EIS at various temperatures.

\section{Materials \& Experiments}

This section describes two experiments performed to assess the credibility and usability of ACI measurements obtained in under load environments. A preliminary experiment was performed to observe how the ACI spectrum changes when subjected to various constant current loads and to define the limitations of EIS in such conditions. In a follow-up experiment, EIS was performed to evaluate the usability of ACI for temperature determination.

\subsection{Materials Used}

Consumer-grade Samsung Li-ion NMC-based 18650 cells were used for the experiment. The manufacturer's product data sheet defined a rated capacity of 3.0Ah. Individual battery cells were connected via high current battery holders. In order to perform EIS testing while simultaneously subjecting cells to constant current loads, ReJoule's patent pending EIS analyzer was paired with a battery cycler. The battery cycler, also created by ReJoule, was connected in parallel with the EIS analyzer to provide the necessary direct current (DC) loads. Additionally, throughout all experiments, ambient testing temperatures were regulated with thermal chambers. Two different thermal chambers were used-a Tenney $\mathrm{Jr}$ and a Missimer. In all EIS experiments, settings were kept constant: galvanostatic mode with a $0.3 \mathrm{~A}$ perturbation amplitude, 10 frequencies per decade, and a frequency range from $1000 \mathrm{~Hz}$ to $0.79 \mathrm{~Hz}$.

\subsection{EIS Under Load Experimental Set Up}

A set of EIS under load experiments were performed to assess the validity of ACI measurements taken while a battery is also under a constant current load. Testing required a means of providing a DC bulk current while simultaneously performing galvanostatic EIS. In each experiment, a custom battery cycler and custom EIS analyzer were each programmed to perform successive EIS tests while the battery was under the following conditions: at rest, discharging, and charging. An idle period of two minutes was used to allow for minimal relaxation of the battery between the discharge and charge pulses. Given the EIS settings defined in Section 3.1, each EIS test required roughly 90 
seconds to complete. Throughout testing, the cell voltage was kept within a range of $3.4 \mathrm{~V}$ to $4.1 \mathrm{~V}$ to reduce measurement error and to obtain comparable ACI spectra. Within this range, ACI profiles are expected to remain relatively constant, which is ideal for later comparisons of the results (17).

Experiments were repeated in different test temperature settings ranging from $5^{\circ} \mathrm{C}$ to $35^{\circ} \mathrm{C}$ in $10^{\circ} \mathrm{C}$ increments. Prior to each experiment, the battery was left at rest for at least one hour at the testing temperature to ensure a uniform cell temperature. Additionally, multiple constant current rates were tested at each temperature. Charge rates (C-rates) of $\mathrm{C} / 5, \mathrm{C} / 3,2 \mathrm{C} / 3,1 \mathrm{C}$, and $4 \mathrm{C} / 3$ were tested during charging conditions and discharge rates (D-rates) of $\mathrm{D} / 5, \mathrm{D} / 3,2 \mathrm{D} / 3,1 \mathrm{D}$, and $4 \mathrm{D} / 3$ were tested during discharging conditions.

\subsection{Variable Temperature EIS Experiment}

A follow-up experiment was performed to investigate the usability of EIS under load measurements in estimating the internal cell temperature of a battery subjected to extreme load profiles. In this variable temperature EIS experiment, the battery cycler was connected again in parallel with the EIS analyzer, but EIS tests were performed continuously throughout testing, asynchronous to the DC load current supplied by the battery cycler. The DUT was subjected to large (3.3D) current pulses in order to induce a rise in internal cell temperature. Three negative temperature coefficient (NTC) temperature sensors were adhered to the cell externally with thermally conductive putty as shown in figure 3 for later reference comparisons. Tested load profiles consisted of $10 \mathrm{~A}$ discharge pulses lasting for three, five, and seven minutes each. Cells were initially charged using a constant current / constant voltage $(\mathrm{CC} / \mathrm{CV})$ method to $4.2 \mathrm{~V}$ with a $\mathrm{CC}$ current of $1.5 \mathrm{~A}(\mathrm{C} / 2)$ and a $\mathrm{CV}$ cutoff current of $150 \mathrm{~mA}$. All tests were performed at an ambient temperature of $25^{\circ} \mathrm{C}$, and cells were soaked at the testing temperature for at least one hour prior to introducing the load profile. Additionally, EIS testing was performed throughout the load profile and for an additional 40 minutes after the load profile was concluded.

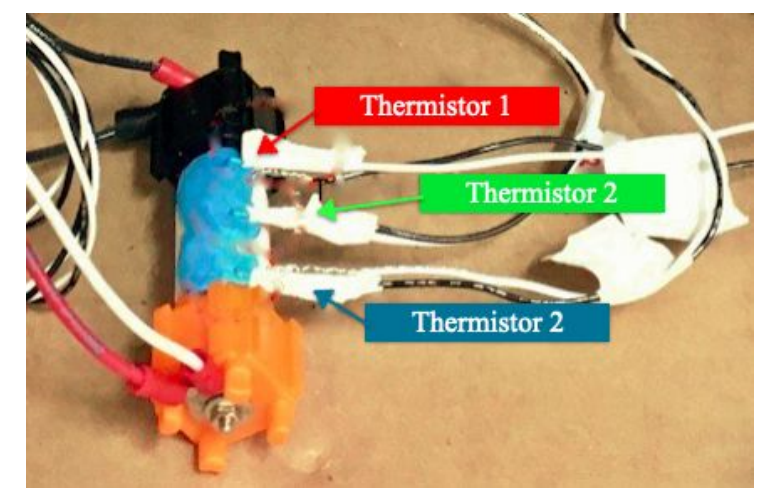

Figure 3. Battery under load test setup and NTC thermistor placement. 


\section{Results}

\subsection{EIS Under Load Results}

ACI spectra gathered during the EIS under load experiment at $5^{\circ} \mathrm{C}$ are shown in the Nyquist plot in figure 4. There is a noticeable difference between ACI spectra taken under load during charging/discharging and the spectrum taken at rest. In both under load ACI spectra, deviations from the ACI spectrum at rest tend to increase as the impedance extends further out in the positive real direction. This deviation, or error, is measured at each frequency. The percentage of the root mean square error (RMSE) at each frequency is calculated and then normalized by the width of the semi-circle in the ACI. The dotted line in figure 4 marks the threshold or frequency limit $\left(f_{\text {lim }}\right)$ beyond which error exceeds $1 \mathrm{e}^{-4} \Omega$.

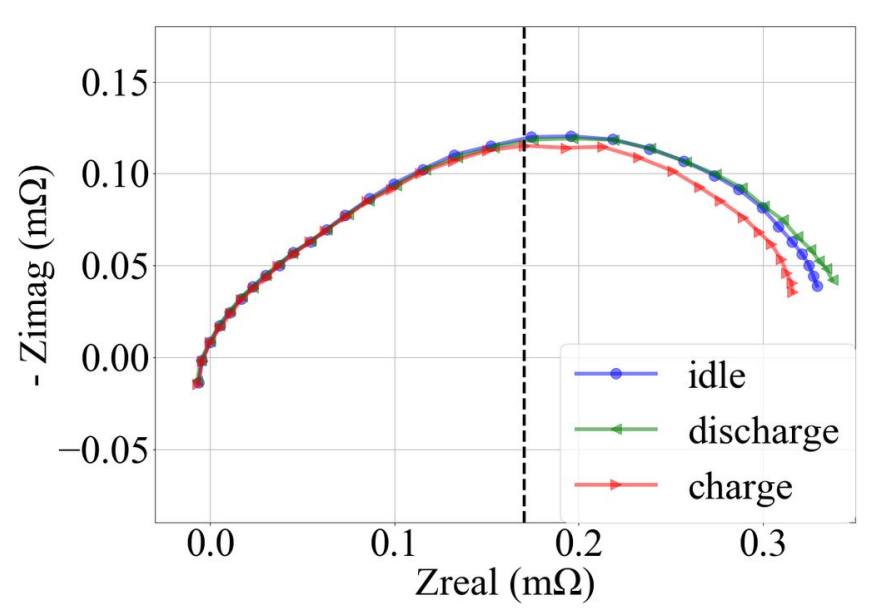

Figure 4. Normalized EIS under load at $5^{\circ} \mathrm{C}$ taken under idle, discharge $(\mathrm{D} / 5)$, and charge $(\mathrm{C} / 5)$ conditions. Dotted line marks the point at which EIS spectra for the charge condition diverges. The $f_{\text {lim }}$ is $19.95 \mathrm{~Hz}$ for the charge (dotted line) and $1.58 \mathrm{~Hz}$ for the discharge.

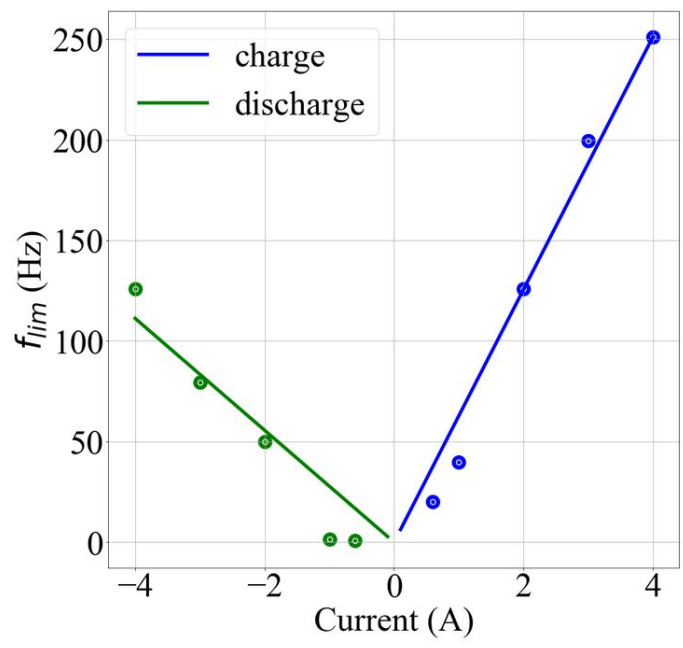

Figure 5. $f_{\text {lim }}$ at different current loads at $5^{\circ} \mathrm{C}$. 
The $f_{\text {lim }}$ was calculated for each tested current rate and plotted above in figure 5 . As the current magnitude increases in both the charge and discharge directions, $f_{\text {lim }}$ can be approximated through a linear regression with relatively high accuracy. This process was repeated across all temperature settings tested in the EIS under load experiment. This linearized relationship can be summarized by equation 2, where $I_{\text {load }}$ represents the current magnitude and $\psi$ in units of $\mathrm{Hz} / \mathrm{A}$ corresponds to the rate at which $f_{\text {lim }}$ changes with $I_{\text {load }}$.

$$
f_{\text {lim }}=\psi I_{\text {load }}
$$

The resulting $\psi$ rates across the tested temperature range are shown in figure 6 , for each of the charge and discharge conditions. At colder temperatures, the amount of anticipated usable ACI information quickly decreases as larger current loads are applied. Alternatively, higher temperatures prove to be more resilient to increasing current loads, as $\psi$ remains relatively low for temperatures at and $25^{\circ} \mathrm{C}$. Additionally, at temperatures $\leq 15^{\circ} \mathrm{C}$ and under, $\psi$ tends to be larger during charging, which is opposite of the behavior at $\geq 25^{\circ} \mathrm{C}$.

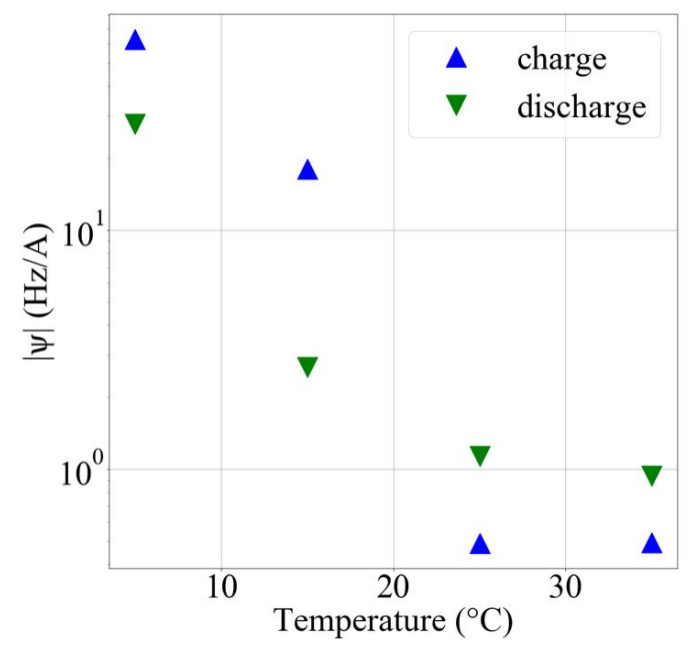

Figure 6. Absolute values of $\psi$ for both charge and discharges at different temperatures.

Overall, an empirical model is determined for $f_{\text {lim }}$ as a function of the temperature and the current load $I_{\text {load }}$ through the variable $\psi$. This threshold is later used to validate ACI measurements prior to estimating internal cell temperature in section (4.2).

\subsection{Variable Temperature EIS Results}

The results of the variable temperature EIS experiment described in section (3.3) are shown in figure 7. The time series current and voltage data are shown indicating periods of extreme conditions during which spikes in both the measured and estimated temperature curves can be seen. The aim is to evaluate the accuracy of an internal cell temperature estimation based on EIS data gathered under loaded conditions. A methodology outlined by Raijmakers et al. is used to approximate internal cell temperature using non-zero intercept frequency (NZIF) on ACI measurements (19). The 
NZIF approach attempts to highlight trends in Nyquist plots due to temperature changes, as previously shown in figure 2. ACI spectra gathered through the continuous EIS tests were first trimmed based on the calculated $f_{\text {lim }}$ as defined in equation 2 prior to performing the NZIF analysis. The resulting internal cell temperature estimation is shown below in figure 7. An average of the three external temperature sensors is also shown and serves as a reference for the temperature behavior.
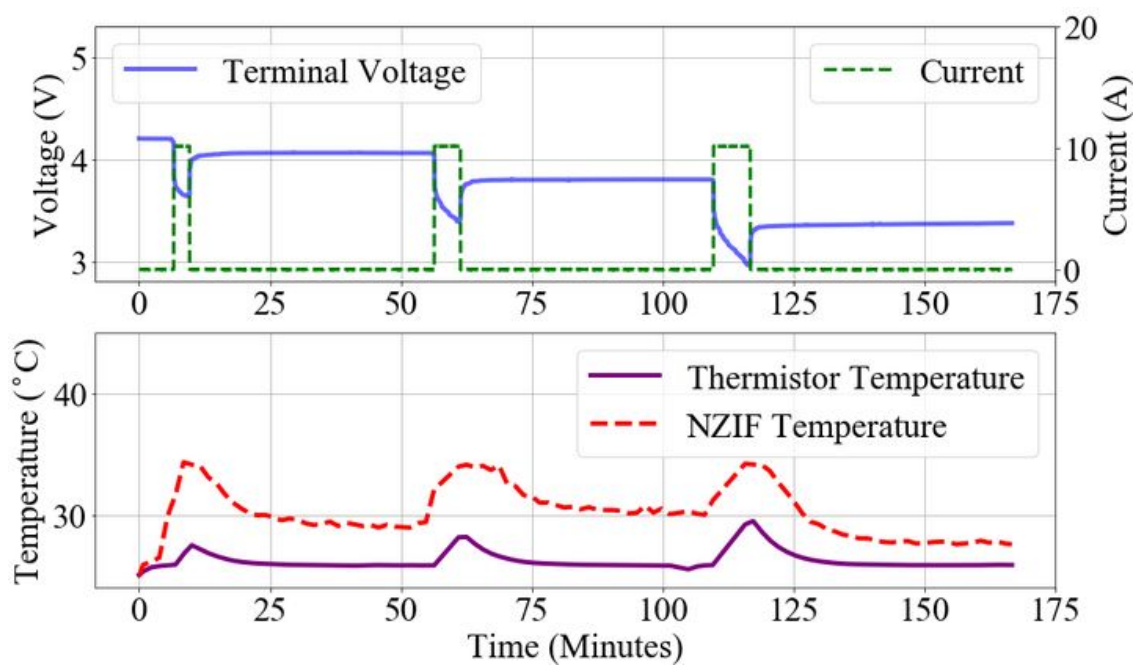

Figure 7. Thermistor and NZIF cell-temperature measurements taken under the plotted current load conditions.

Although the measured thermistor temperature is significantly lower than the NZIF temperature prediction, this does not discredit the accuracy of the NZIF estimation. Surface temperature is expected to remain cooler through the extreme current events as there is sufficient air being circulated within the thermal chamber throughout testing.

\section{Discussion}

When performing EIS on a cell that is in loaded conditions, there are two primary concerns: 1) whether the measurements being taken are accurate and 2) whether the electrochemical interpretation related to the results is compromised. These EIS measurements may yield inaccurate results due to changes in the common-mode voltage $V_{C M}$ because of the manner in which the raw sine wave signals are typically analyzed. When subject to a current load, batteries are charged or discharged, resulting in changes to the $V_{C M}$, even if the common-mode current remains stable as shown in figure 8 . Additionally, as ACI spectra are used to analyze electrochemical phenomena within the battery, it is imperative to consider how these phenomena may be influenced by the introduction of a DC load. Both the electrochemical stability and the measurement accuracy are discussed in the following sections. 


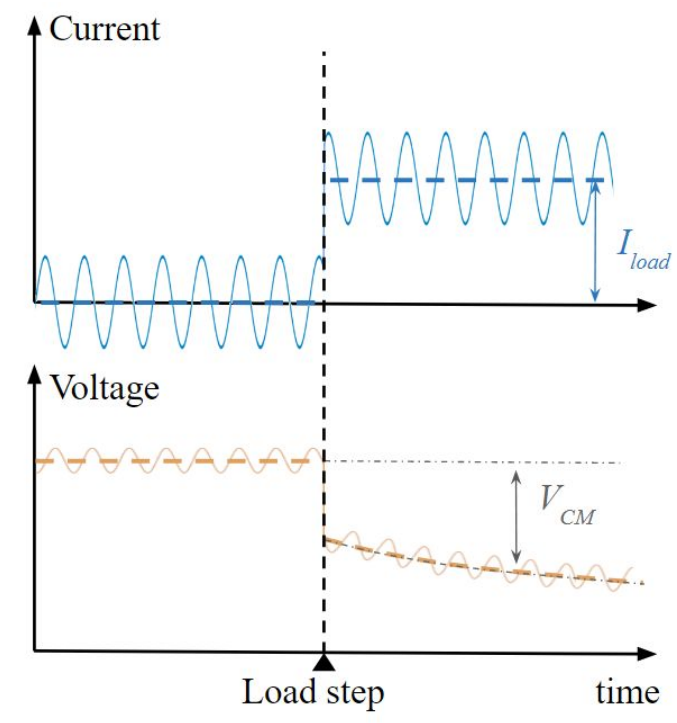

Figure 9. Battery current and voltage EIS signals during an $I_{\text {load }}$ discharge step. $V_{C M}$ shifts as the battery is discharged (values not shown to scale).

\subsection{ACI Deviation due to Measurement Error}

Prior to the experiments, the anticipated measurement concerns were simulated using ideal sine waves and previously measured stable ACI spectra. A major concern in signal processing is the ability for changes in the DC component of the signal to interfere with the AC measurement. This is further exacerbated with EIS, as the AC current and voltage signals must be kept small to maintain system linearity. Therefore, when subject to a constant DC load, the changes in $V_{C M}$ were expected to be the primary source of measurement error. Additionally, larger changes in $V_{C M}$ will yield an even higher $f_{\text {lim }}$, resulting in less usable ACI information. This can be seen in figure 10, where the effects of different load currents were simulated by superimposing ideal voltage sine waves on varying sloped signals.

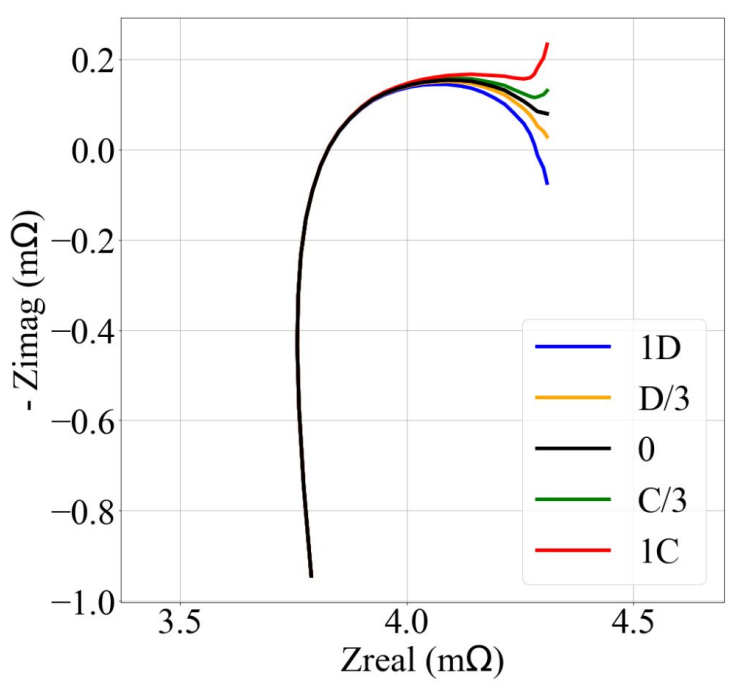

Figure 10. Theoretical ACI spectra vs various current loads. 
The results show that at higher $I_{\text {load }}$, deviation from the stable reference spectra increases. This is consistent with the previous assumption-larger changes in $V_{C M}$ yield higher $f_{\text {lim }}$ - since higher current rates lead to larger changes in $V_{C M}$ as the battery is more rapidly charged or discharged. For this reason, the battery voltage was kept between $3.4 \mathrm{~V}$ and $4.1 \mathrm{~V}$ during experimentation, as the $V_{C M}$ is expected to have a low rate of change with respect to change in SOC. This can be seen in the state-of-charge vs. open circuit voltage (SOC-OCV) curve shown in figure 11, which can be used to estimate the $V_{C M}$ behavior at different SOCs.

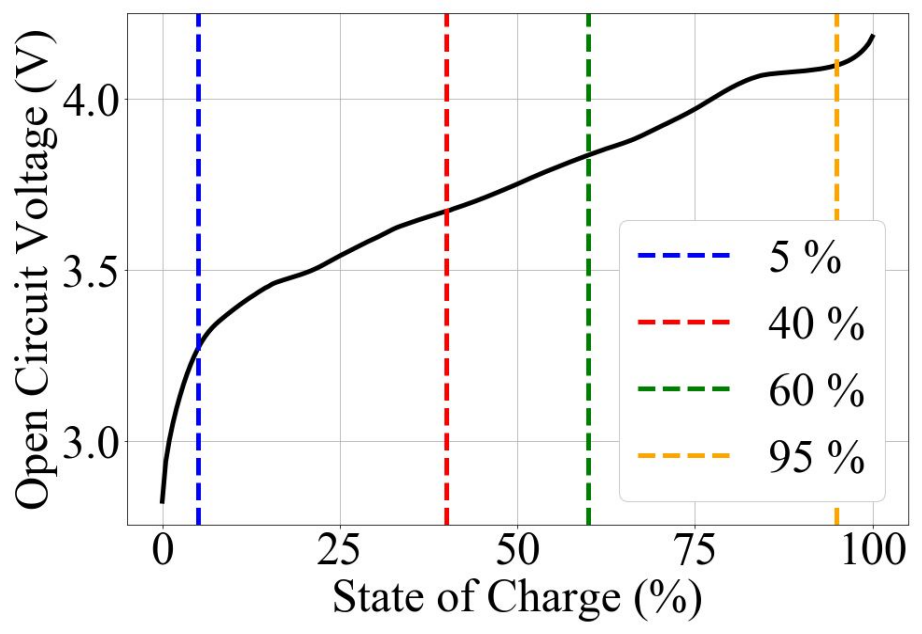

Figure 11. SOC-OCV curve for batteries tested in experiments. Dashed lines identify SOC's used in figure 12.

A major drawback of many EIS analyzers on the market today is that time series current and voltage signals are often hidden from the user. Many EIS tools simply assume system linearity, causality and stability criteria are met and only output the resulting ACI information. Therefore in unstable conditions, such as when the battery is under load, filtering out the effects of the changing $V_{C M}$ is difficult. However, preliminary simulations were also performed where ideal sine waves were superimposed at different places along the OCV curve shown in figure 11, as marked by the dashed lines. A linear approximation of the $V_{C M}$ behavior was then filtered out prior to impedance computation. The results shown on figure 12 (right panel) are promising, as the deviation among the plots is significantly reduced when compared to figure 12 (left panel). Performance is particularly good at SOC's where the SOC-OCV curve is more linear. Additionally, this type of simple correction could be applied in real-time assuming there is also real-time DC voltage sensing to accompany the AC measurements. Although this type of correction is fairly crude, it can potentially allow for valid ACI measurements in more extreme conditions. Furthermore, if better tracking of $V_{C M}$ behavior is available, EIS testing can be performed over an even wider voltage range at nonlinear sections of the curve. 

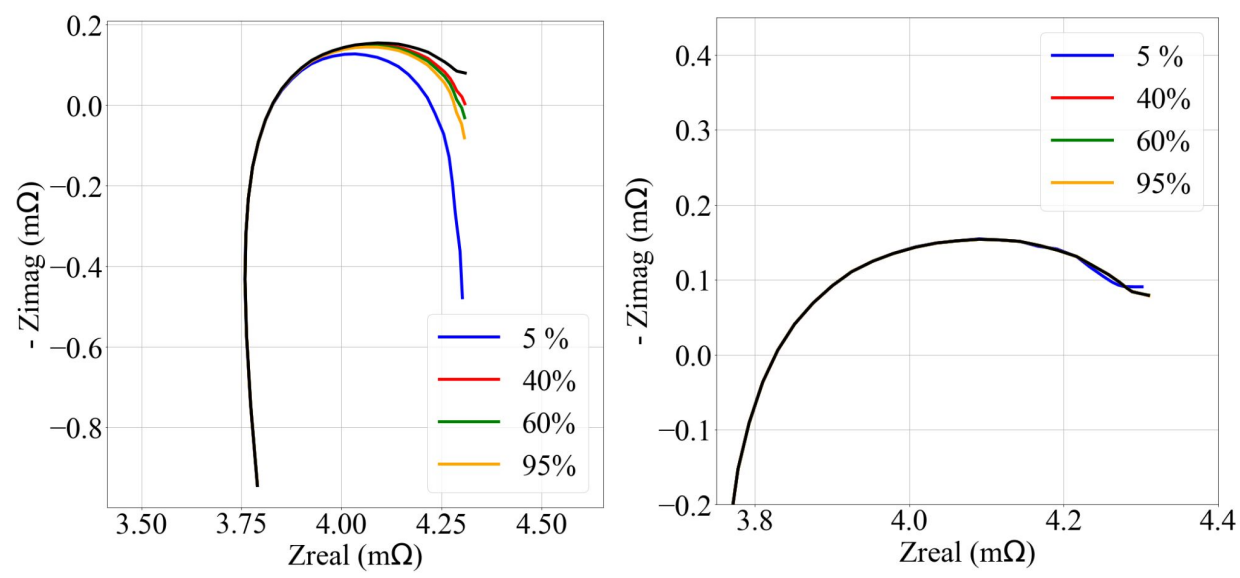

Figure 12. (left) Theoretical EIS under 1C load at varying SOC, (right) Demonstration of Linear Correction (zoom).

\subsection{Electrochemical Sources of Deviations}

Given the EIS under load conditions, the relationship between ACI measurements and the electrochemical phenomena described in section 2 must be reevaluated. While diffusion still takes place when under load, a migration phenomena, due to an electric potential gradient, is also taking place. Therefore, the corresponding low frequency ACI measurements can no longer be used to directly analyze the diffusion and intercalation/deintercalation phenomena independently.

Current flowing through the battery generates heat because of the cell's internal resistance. However, the internal resistance changes with respect to temperature (20). This is illustrated in figure 7 where both the measured and estimated temperatures tend to stabilize after being subjected to a current pulse over a short period of time.

While temperature influences the ACI measurement as shown in figure $2, f_{\text {lim }}$ also increases at lower temperatures. Thus, $|\psi|$ is lower at higher temperatures along with internal resistance. Additionally, as seen in figure 6, a non-symmetrical behavior is observed in $|\psi|$ when comparing the effects of charging and discharging.

As mentioned earlier, EIS measurements are very sensitive to changes in temperature. Even though the thermodynamics of Li-ion cells are complex and consist of both liquid electrolyte and solids in various phases, the discharge processes are exothermic $(\Delta \mathrm{G}<0)$ (21). This implies that batteries generate extra heat during discharging, all of which influences $f_{\text {lim }}$. Thus, at high temperatures $\geq 25^{\circ} \mathrm{C},|\psi|$ is higher during discharging than it is during charging as seen in figure 6. At lower temperatures, the inversion can be attributed to higher thermodynamic instability and additional stresses on the battery's material structure than is experienced during discharging. This compromises ACI measurements and results in $|\psi|$ being higher during charging than during discharging at lower temperatures $\leq 15^{\circ} \mathrm{C}$. 


\subsection{Application of EIS Under Load for Temperature Determination}

A main concern of the battery industry, especially in EV applications, is maintaining a constant battery temperature since batteries have an optimal operating temperature-range. Therefore, it is important to have a good approximation of cell temperature. Industry relies on external temperature sensors. This adds cabling, long latency, and inaccuracy in temperature readings. Considering the importance of temperature for prolonging a battery's life in ESS and EV applications, EIS was utilized here to approximate internal cell temperature.

The empirical model in section (4.1) for the $f_{\text {lim }}$ was used to identify the usable part of an ACI measurement and input it into the NZIF temperature estimation (19). As expected, in figure 7 the NZIF internal cell temperature prediction exceeds the averaged measured thermistor readings. Also, the trend in NZIF internal cell temperature approximation is consistent with behavior observed in thermistor measurements.

An inconsistency in the results can be observed at the end of the last current pulse in figure 7 , as the temperature of the cell is expected to increase after each high current event. However, the internal cell temperature estimation after the final current event settles at a temperature lower than the estimation prior to the final discharge pulse. This is likely due to a violation of the voltage range defined in section $3.2(3.4 \mathrm{~V}-4.1)$. Despite this discrepancy, the methodology used for approximating internal cell temperature holds. Future work will be done to account for this discrepancy.

Overall, the variable temperature EIS experiment validates the use of a real-time temperature estimation using EIS under load. This method can be used for more proactive temperature determination in various applications.

\section{Conclusion}

ACI measurements on batteries are already used as a powerful tool for assessing a battery's SOH. However, acquiring intelligible ACI measurements of a battery under load (i.e., during discharging or charging) is challenging. In this work, ACI measurements were not only taken under load but also used to approximate the internal temperature of a battery. These are promising findings for monitoring and indeed mitigating a battery's degradation using more precise thermal management for batteries of all kinds, not just Li-ion. 


\section{Appendix}

Appendix A - SOC deviation

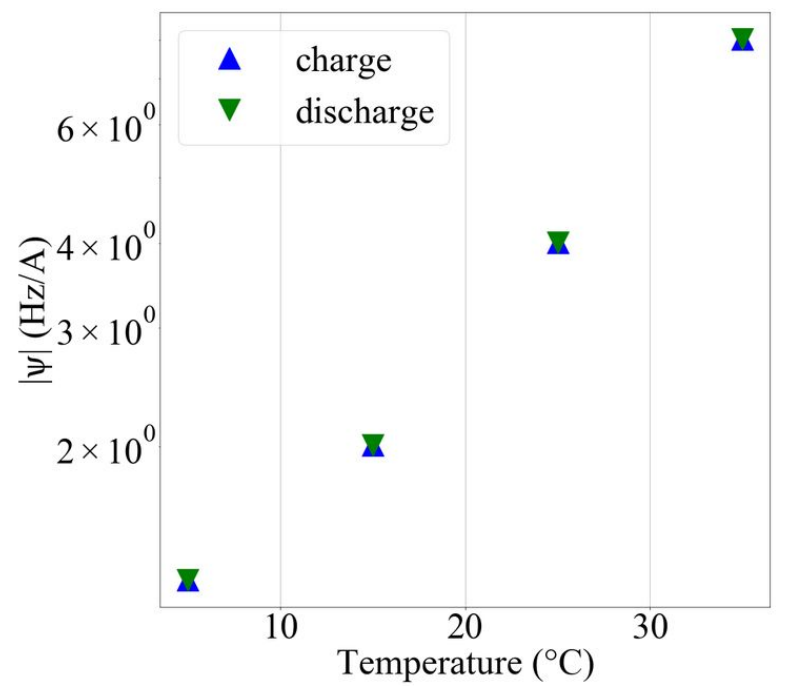

Figure 13. absolute $\psi$ values for the theoretical SOC deviation

The SOC deviation could not be quantified using the deviation detection threshold. However, the deviation due to the SOC exists and can still be quantified when lowering the deviation detection threshold for the frequency limit down to $1 \mathrm{e}^{-5} \Omega$. The $f_{\text {lim }}$ was calculated by superimposing ideal voltage response signals over the semi-linear zone of the SOC-OCV curve from $3.4 \mathrm{~V}$ to $4.1 \mathrm{~V}$. Figure 13 . The results in figure 10 show the charge and discharge points are overlapping, which is expected as the theoretical deviations are symmetrical. The effect of this deviation goes against the trend that was determined in figure 6 . The $\psi$ values cannot be compared to the ones in figure 6 as the threshold is different and the measured deviation is different. It is to be noted that the OCV-curve depends on the battery chemistry and the semi linear region is subject to changes. However, for battery chemistries with a relatively flat OCV-curve such as Lithium Iron Phosphate (LFP), there is even less deviation due to the SOC deviation.

\section{$\underline{\text { Appendix B }-\Psi \text { values }}$}

TABLE I. Real deviation $\Psi$ values used in figure 6 with forced $(0 \mathrm{~A}, 0 \mathrm{~Hz})$ point, threshold at $1 \mathrm{e}^{-4} \Omega$..

\begin{tabular}{ccc}
\hline Temperature / state / type & $\boldsymbol{\Psi}(\mathbf{H z} / \mathbf{A})$ & $\mathbf{R}^{2}$ \\
\hline $5^{\circ} \mathrm{C}$ / charge / Real & 62.81 & 0.9920 \\
$15^{\circ} \mathrm{C}$ / charge / Real & 18.473 & 0.8644 \\
$25^{\circ} \mathrm{C}$ / charge / Real & 0.4896 & 0.9273 \\
$35^{\circ} \mathrm{C}$ / charge / Real & 0.4932 & 0.9434 \\
\hline $5^{\circ} \mathrm{C} /$ discharge / Real & -27.805 & 0.9513 \\
$15^{\circ} \mathrm{C}$ / discharge / Real & -2.6696 & 0.7807 \\
$25^{\circ} \mathrm{C} /$ discharge / Real & -1.1312 & 0.9800 \\
$35^{\circ} \mathrm{C}$ / discharge / Real & -0.9371 & 0.6256 \\
\hline
\end{tabular}


TABLE II. Theoretical deviation $\Psi$ values used in figure 12 , threshold at $1 \mathrm{e}^{-5} \Omega$.

\begin{tabular}{ccc}
\hline Temperature / state / type & $\boldsymbol{\Psi}(\mathbf{H z} / \mathbf{A})$ & $\mathbf{R}^{2}$ \\
\hline $5^{\circ} \mathrm{C} /$ (dis)charge / Theoretical & $(-) 1.2707$ & 0.9974 \\
$15^{\circ} \mathrm{C} /$ (dis)charge / Theoretical & $(-) 2.0107$ & 0.9973 \\
$25^{\circ} \mathrm{C} /$ (dis)charge / Theoretical & $(-) 4.0095$ & 0.9973 \\
$35^{\circ} \mathrm{C} /$ (dis)charge / Theoretical & $(-) 8.0033$ & 0.9973 \\
\hline
\end{tabular}

\section{Acknowledgements}

The authors would like to thank the National Science Foundation for funding a portion of this research (NSF award \#1842957).

\section{References}

1. S. J. Harris, D. J. Harris, C. Li. J. Power Sources 342 (2017)

2. D. Devaux, H. Leduc, P. Dumaz, M. Lecuyer, M. Deschamps, and R. Bouchet. Frontiers in Energy Research. 7 (2020).

3. G. L. Plett. J. Power Sources. 134 (2004).

4. F. Zhang, M. M. U. Rehman, H. Wang, Y. Levron, G. Plett, R. Zane, and D. Maksimović. IEEE COMPEL. (2015)

5. B. Rajabloo, A. Jokar, M. D'esilets, and Marcel Lacroix. J. Electrochem. Soc., 164 (2) A99-A105 (2017)

6. V. R. Subramanian, V. Boovaragavan, and V. D. Diwakar. Electrochem, Solid-State Lett, 10 (11) A255-A260 (2007)

7. Y. H. Lui, M. Li, M. Sadoughi, C. Hu, S. Hu. American Soc. Mechanical Engineers. 44th Design Automation Conference (2018).

8. S. F. Schuster, M. J. Brand, C. Campestrini, M. Gleissenberger, and A. Jossen, J. Power Sources, 305 (2016).

9. C. Pastor-Fernández, K. Uddin, G. H. Chouchelamane, W. D. Widanage, and J. Marco. J. Power Sources, 360 (2017).

10. U. Westerhoff, T. Kroker, K. Kurbach, and M. Kurrat. J. Energy Storage. 8 (2016).

11. A.Wang, Y. Zhang, and H. Zuo. Mathematical Problems in Engineering. 2019 (2019)

12. C. T. Love, M. B. V. Virji, R. E. Rocheleau, and K. E. Swider-Lyons. J. Power Sources 266 (2014).

13. E. Din, C. Schaef, K. Moffat and J. T. Stauth. IEEE Trans. on Power Electronics, 32(7) (2017).

14. M. Kuipers, H. Zappen, P. Schröer, and D. Sauer. Advanced Battery Conference (2019). 
15. Z. Gong, Z. Liu, Y. Wang, K. Gupta, C. da Silva, T. Liu, Z. H. Zheng, W. P. Zhang, J. P. M. van Lammeren, H. J. Bergveld, C. H. Amon, O. Trescases. IEEE APEC. (2018)

16. J.-P. Diard, B. Le Gorrec, and C. Montella, J. Power Sources. 70, 1 (1998).

17. V. J. Ovejas and A. Cuadras. Batteries. 4(3),43. (2018).

18. W. Choi, H. Shin, J. M. Kim, J. Choi, and W. Yoon, J. Electrochemical Science and Technology. 11(1)1-13 (2020).

19. L. H. Raijmakers, D. L. Danilov, J. P. M. Van Lamemeren, T. J. G. Lammers, H. J. Bergveld, and P. H. L. Notten, IEEE Trans. of Industrial Electronics. 63(5) (2016).

20. A. A. Hussein. IEEE APEC. (2015)

21. J. A. Osara, and M. D. Bryant. Inventions. 4(3) (2019) 